CLINICAL STUDY

\title{
Increased hypothalamic-pituitary-adrenal drive is associated with decreased appetite and hypoactivation of food-motivation neurocircuitry in anorexia nervosa
}

\author{
Elizabeth A Lawson*, Laura M Holsen ${ }^{1,2, *}$, Rebecca DeSanti, McKale Santin, Erinne Meenaghan, \\ David B Herzog ${ }^{3}$, Jill M Goldstein ${ }^{1,2}$ and Anne Klibanski \\ Neuroendocrine Unit, Massachusetts General Hospital and Harvard Medical School, 55 Fruit Street, Bulfinch 457-D, Boston, Massachusetts 02114, \\ USA, ${ }^{1}$ Division of Women's Health, Department of Medicine, and ${ }^{2}$ Department of Psychiatry, Brigham and Women's Hospital and Harvard Medical School, \\ Boston, Massachusetts 02120, USA and ${ }^{3}$ Department of Psychiatry, Massachusetts General Hospital and Harvard Medical School, Boston, Massachusetts \\ 02114, USA
}

(Correspondence should be addressed to E A Lawson; Email: ealawson@partners.org)

*(E A Lawson and L M Holsen are co-first authors)

\begin{abstract}
Objective: Corticotrophin-releasing hormone (CRH)-mediated hypercortisolemia has been demonstrated in anorexia nervosa (AN), a psychiatric disorder characterized by food restriction despite low body weight. While CRH is anorexigenic, downstream cortisol stimulates hunger. Using a food-related functional magnetic resonance imaging (fMRI) paradigm, we have demonstrated hypoactivation of brain regions involved in food motivation in women with $\mathrm{AN}$, even after weight recovery. The relationship between hypothalamic-pituitary-adrenal (HPA) axis dysregulation and appetite and the association with food-motivation neurocircuitry hypoactivation are unknown in AN. We investigated the relationship between HPA activity, appetite, and food-motivation neurocircuitry hypoactivation in AN.

Design: Cross-sectional study of 36 women (13 AN, ten weight-recovered AN (ANWR), and 13 healthy controls (HC)).

Methods: Peripheral cortisol and ACTH levels were measured in a fasting state and 30, 60, and $120 \mathrm{~min}$ after a standardized mixed meal. The visual analog scale was used to assess homeostatic and hedonic appetite. fMRI was performed during visual processing of food and non-food stimuli to measure the brain activation pre- and post-meal.

Results: In each group, serum cortisol levels decreased following the meal. Mean fasting, 120 min postmeal, and nadir cortisol levels were high in AN vs HC. Mean postprandial ACTH levels were high in ANWR compared with $\mathrm{HC}$ and AN subjects. Cortisol levels were associated with lower fasting homeostatic and hedonic appetite, independent of BMI and depressive symptoms. Cortisol levels were also associated with between-group variance in activation in the food-motivation brain regions (e.g. hypothalamus, amygdala, hippocampus, orbitofrontal cortex, and insula).

Conclusions: HPA activation may contribute to the maintenance of AN by the suppression of appetitive drive.
\end{abstract}

European Journal of Endocrinology 169 639-647

\section{Introduction}

Anorexia nervosa (AN) is a psychiatric disorder characterized by food restriction despite extremely low weight. Corticotrophin-releasing hormone (CRH)mediated hypercortisolemia has been described in these patients, presumably due to the stress of chronic starvation $(1,2,3,4,5,6,7)$. However, hypothalamicpituitary-adrenal (HPA) dysregulation may persist in AN after weight gain, suggesting that this pathway may be involved in disease pathogenesis $(8,9)$. While CRH is anorexigenic, signaling satiety, cortisol excess, such as in Cushing's disease or exogenous glucocorticoid exposure, results in increased appetite and weight gain $(10,11,12)$. This may be related to a direct effect of cortisol on the appetite-regulating regions of the brain, or indirect effects through inhibition of the anorexigenic hypothalamic hormone CRH or modulation of other appetite-regulating hormones $(11,13,14)$. We have previously shown that nocturnal cortisol levels are associated with the severity of disordered eating psychopathology in women across the weight spectrum, independent of BMI (15). Using a food-related functional magnetic resonance imaging (fMRI) 
paradigm, we have also reported hypoactivation of food-motivation neurocircuitry in women with AN compared with healthy women, even after recovery (16). In the aforementioned study, women with AN reported lower subjective appetite levels than healthy women (16). Whether HPA dysregulation is associated with differences in appetite or hypoactivation of brain circuits involved in food motivation in AN is unknown.

In this study, we use a previously validated fMRI paradigm with food and non-food visual stimuli and endocrine assessment during fasting and following a standardized mixed meal to examine the link between HPA secretory abnormalities and appetite and brain circuitry deficits in AN (16). Comorbid psychiatric disorders, including depression, are common in AN, and there is overlap between brain regions (e.g. hypothalamus, amygdala, hippocampus, and insula) involving stress and food-motivation pathways. Importantly, modulation of appetite and feeding behavior by HPA hormones is specifically potentiated through dense expression of receptors (CRF1, CRF2, and GR) in these same limbic and paralimbic regions: hypothalamic nuclei (ventromedial hypothalamus; paraventricular nucleus), amygdala, hippocampus, nucleus accumbens, and insula $(17,18,19,20,21)$. We hypothesized that the relationships between HPA hormone levels and measures of appetite and brain activation would be independent of depressive symptoms.

\section{Subjects and methods}

\section{Subjects}

We studied 36 women between 18 and 28 years of age: 13 with AN, ten who had recovered from AN (ANWR), and 13 normal weight healthy controls (HC). Subject demographics, validation of the fMRI paradigm, and cortisol levels have been previously published $(16,22$, 23). In this paper, we investigated the relationship between HPA activation and appetite and foodmotivation neurocircuitry. All study participants were recruited from the community through advertisements and were referrals from healthcare providers.

Subjects were excluded if they had any contraindication to MRI such as an implanted medical device, significant orthopedic hardware, or severe claustrophobia. Additional exclusion criteria included active abuse of drugs or alcohol, use of hormones or medications known to affect hormone levels (including estrogen) within 8 weeks of the study visit, use of depot medroxyprogesterone within 6 months, diabetes mellitus, history of gastrointestinal tract surgery, pregnancy or breastfeeding within 8 weeks of the study, and hematocrit $<30 \%$ or hemoglobin $<10 \mathrm{~g} / \mathrm{dl}$.

Subjects met the diagnostic criteria for AN with the Structured Clinical Interview for DSM Disorders-IV (SCID), including intense fear of gaining weight, evidence of body image disturbance, significantly low body weight (operationalized as $<85 \%$ of ideal body weight (IBW) as determined by the 1983 Metropolitan Life tables), and amenorrhea for at least 3 consecutive months (24). AN subjects who reported more than one binge and one purge episode per month in the 3 months preceding the study were excluded. Subjects with a history of psychosis by SCID were also excluded.

ANWR subjects were between 90 and $110 \%$ of IBW and were required to have regular menstrual cycles and stable weight for at least 6 months prior to the study. ANWR subjects met a diagnosis of AN by DSM-IV criteria other than amenorrhea, as assessed by SCID, in the past. The recovered subjects had not exercised more than $10 \mathrm{~h} /$ week and had not run more than 25 miles/week in the 3 months preceding the study.

HC were between 90 and 110\% of IBW and reported regular menstrual cycles. HC had no history of amenorrhea, no acute or chronic illnesses, and no history of a psychiatric disorder (including an eating disorder) as assessed by SCID. HC were excluded if they had exercised more than $10 \mathrm{~h} /$ week or ran more than 25 miles/week in the 3 months preceding the study.

\section{Methods}

This study was approved by the Partners Human Research Committee. Written informed consent was obtained from all subjects prior to conducting any procedures. All subjects were admitted to the Massachusetts General Hospital (MGH) Clinical Research Center for an outpatient screening visit and to the MGH Clinical Research Center and Athinoula A Martinos Imaging Center for a morning, outpatient main visit.

At the screening visit, height, weight, and elbow breadth were measured by research dietitians, blood was drawn for screening laboratory tests, and a comprehensive history and physical exam was performed. Exercise patterns and alcohol intake were assessed. Percent IBW was calculated as above. BMI was obtained by dividing the weight in kilograms by the square of height in meters. Frame size was determined by comparing elbow breadth with race-specific norms derived from the US Health and Nutritional Examination Survey-I (25). The mood episode, psychotic and associated symptoms, mood disorder, anxiety, somatoform, substance abuse, and disordered eating modules of the structured clinical interview for DSM disorders-IV (SCID) were administered in person during the screening visit or over the telephone before the main visit by a trained psychiatric nurse practitioner or psychologist (24).

At the main visit, \%IBW and BMI were reevaluated. A brief medical history was performed. HC and ANWR were presented during the follicular phase of the menstrual cycle (days 1-10). Subjects were asked to fast for $12 \mathrm{~h}$ prior to the visit. Subjects were given a $400 \mathrm{kcal}$ mixed breakfast meal standardized for micro- and 
macronutrient content $(\sim 20 \%$ calories from protein, $20 \%$ from fat, and $60 \%$ from carbohydrates) at $0900 \mathrm{~h}$. The participants selected one of the following options of similar macro- and micronutrient content, provided by the Clinical Research Center bionutritionists: cereal, lowfat milk, yogurt, and wheat germ; or minibagel with peanut butter, nonfat lactaid milk, or craisins. Participants were asked to eat the entire meal over a 15 min interval. Upon completion of the meal, the bionutrition staff weighed the meal to determine exact caloric intake. Blood was drawn at four different time points throughout the morning for hormone levels: a fasting blood draw obtained immediately before the standardized mixed meal, and blood draws at 30, 60, and $120 \mathrm{~min}$ after the meal. Subjective appetite was assessed using the visual analog scale. Depressive symptoms were assessed using the Beck Depression Inventory 2 (BDI 2). fMRI using a food-related paradigm was performed before and after the meal (see below).

\section{Biochemical analysis}

Plasma samples were immediately placed on ice. Serum and plasma samples were stored at $-80{ }^{\circ} \mathrm{C}$ until analysis. Serum cortisol levels were measured using a chemiluminescent immunoassay from Beckman Coulter (Fullerton, CA, USA). The intra-assay coefficient of variation (CV) was 4.4-6.7\%, the inter-assay $\mathrm{CV}$ was $6.4-7.9 \%$, and the sensitivity was $0.4 \mu \mathrm{g} / \mathrm{dl}$. Plasma ACTH levels were measured using an IRMA assay from DiaSorin, Inc. (Stillwater, MN, USA). The intra-assay CV was $3.5-4.8 \%$, the inter-assay $C V$ was $3.2-5.7 \%$, and the lowest reportable value was $1.5 \mathrm{pg} / \mathrm{ml}$. Area under the curve (AUC) was calculated using the trapezoidal method.

\section{Assessment of appetite}

Visual analog scales, a reliable and widely used method to assess appetite (26), were administered during the fasting state and following the mixed meal. Subjects were asked to answer questions about appetite by making a mark on a line with extremes on either end indicating how they felt at that moment. For example, in response to the question, 'How hungry are you?', they marked their degree of current hunger between the two extremes, 'I am not hungry at all', on the left and 'I have never been more hungry', on the right. Scores were calculated by measuring the distance from the left side of the line.

\section{Statistical analysis}

JMP Statistical Discoveries (version 9.0; SAS Institute, Inc., Cary, NC, USA) was used for statistical analyses. Hormone levels were not normally distributed and were log-transformed before analysis. Clinical characteristics, hormone levels, and visual analog scores were compared using the overall ANOVA; variables that were significantly different were then compared by Fisher's least significant difference test. Within group comparisons of hormone levels and appetite at different time points were made using the two-sided paired $t$-test. Linear regression analyses were used to investigate the relationships between cortisol levels and subjective appetite measures. Multivariate least-square analyses were constructed to control for potential confounders. Statistical significance was defined as a two-tailed $P$ value $<0.05$. Data are reported as mean \pm s.E.M.

\section{fMRI procedures}

fMRI procedures have been previously validated in this population (16). Briefly, fMRI scanning was performed while subjects viewed high-calorie food stimuli, lowcalorie food stimuli, non-food stimuli, and low-level baseline stimuli in a block design, while participants underwent standard gradient-echo EPI imaging on a Siemens 3T Trio (Malvern, PA, USA).

\section{fMRI data analysis}

fMRI data were analyzed as previously described (16). Data were preprocessed using Statistical Parametric Mapping (SPM8; Wellcome Trust Centre for Neuroimaging at University College London, 2008) and custom routines in MATLAB (Mathworks, Inc., 2000, Natick, MA, USA). Standard preprocessing steps included realignment and geometric unwarping of EPI images using magnetic fieldmaps, correction for bulk-head motion, nonlinear volume-based spatial normalization using the standard brain template from Montreal Neurological Institute (Montreal, Canada), spatial smoothing with a Gaussian filter $(6 \mathrm{~mm}$ full-width at half-maximum), and outlier detection and exclusion (27). Following preprocessing, statistical analysis was performed at the single-subject level. Specific comparisons of interest (high-calorie foods vs objects, separately for pre- and post-meal) were tested using linear contrasts, and SPM maps were created based on these contrasts. Results from the single-subject level were submitted to a second-level random effects analysis. Independent sample $t$-tests were used to compare the size of a particular effect between groups. Clusters were identified within our regions of interest (hypothalamus, amygdala, hippocampus, orbitofrontal cortex (OFC), and anterior insula) in between-group contrasts, significant at $P<0.05$ (uncorrected) and $P<0.1$ (corrected for multiple comparisons within the search volume using voxel-level family-wise error correction).

Anatomic overlays were used on each subject's statistical maps to acquire signal change values across the regions of interest. Values indicated the degree of change in magnetic resonance signal detected between the high-calorie food and object conditions. Average 
percent signal change values (beta weights averaged across all voxels within an anatomical region) were obtained using the REX toolbox for SPM8 (17) and used for brain-hormone general linear model (GLM) analyses. Using PROC MIXED model approach in SAS (version 9.2; SAS Institute, Inc.), the effect of cortisol on the association between group status and brain activity was assessed. The percent change in the estimate for case status when the model was adjusted for the hormone was calculated as the estimate for case status in the univariate model (b1) minus the estimate for case status in the model adjusted for the hormone (b2) together over the univariate estimate (b1) $((b 1-b 2) / b 1)$. Owing to our interest in the mediating effect of cortisol on the case status effect on brain activity, decreases in percent change were of interest, indicating the percent of the case group's effect on brain activity accounted for by the hormone. To examine whether the effects of cortisol on betweengroup differences in brain activity were independent of depressive symptoms, BDI2 scores were entered into the model with cortisol. The percent change in the estimate for case status in the model adjusted for both cortisol and the potential confounder (BDI2) (b3) was compared with the estimate for the case status adjusted solely for the hormone (b2). Again, decreases in percent change (b3 > b2) were of interest, indicating the percent of the case group's effect on brain activity was not confounded by BDI2 scores.

\section{Results}

\section{Subject characteristics}

The mean age of subjects was $22.3 \pm 0.4$ years and did not differ between groups. As per study design, BMI and \%IBW were lower in AN $\left(17.7 \pm 0.3 \mathrm{~kg} / \mathrm{m}^{2}\right.$ and $80.6 \pm 1.3 \%$ respectively) than ANWR (21.9 \pm $0.7 \mathrm{~kg} / \mathrm{m}^{2}$ and $\left.97.7 \pm 3.4 \%\right)$ and $\mathrm{HC}\left(22.5 \pm 0.4 \mathrm{~kg} / \mathrm{m}^{2}\right.$ and $97.2 \pm 1.7 \%)(P<0.0001)$. Mean time since last menstrual period was 50.2 \pm 11.1 months for AN. For ANWR, time since weight recovery was $41.5 \pm 11.1$ months, and time since restoration of menstrual cycles was $42.4 \pm 15.4$ months. All ANWR reported weight stability for at least 12 months and regular menstrual cycles for at least 14 months. Duration of illness did not significantly differ between groups (AN 52.7 \pm 11.2 vs ANWR 43.2 \pm 9.0 months). Three AN and four ANWR reported a remote (at least 14 months before the study) history of purging activity, but none were actively binging or purging. Five AN were taking psychotropic medications: two were taking venlafaxine, one was taking fluoxetine, one was taking a low dose of amphetamine/dextroamphetamine $(5 \mathrm{mg} 24 \mathrm{~h}$ before the scan), and one was taking escitalopram and aripiprazole. Two ANWR were taking psychotropic medications: one was taking fluoxetine and one was taking bupropion and lorazepam. BDI2 scores were higher in AN compared with ANWR and HC (16.8 \pm 3.3 vs $7.7 \pm 2.2$ and $0.8 \pm 0.4, P<0.02$ ), indicating greater severity of depressive symptoms. No subjects smoked cigarettes in the morning of the study period or consumed caffeine within $12 \mathrm{~h}$ of the study period. Hours of sleep the prior night, time since last p.o. intake, and calories consumed at breakfast did not differ between the groups.

\section{Hormone levels}

Hormone levels are presented in Table 1. Mean fasting, $120 \mathrm{~min}$ post-meal, and nadir cortisol levels were higher in AN than HC. In contrast, mean ACTH levels were higher in ANWR than $\mathrm{AN}$ and $\mathrm{HC}$ at 60 min postmeal and $\mathrm{HC}$ at 120 min post-meal. In each group,

Table 1 Hormone levels.

\begin{tabular}{|c|c|c|c|c|c|c|c|}
\hline & \multicolumn{3}{|c|}{ Mean \pm s.E.M. $(p g / m l)$} & \multicolumn{4}{|c|}{$P$ values } \\
\hline & AN & ANWR & $\mathrm{HC}$ & AN vs ANWR & AN vs $\mathrm{HC}$ & ANWR vs $\mathrm{HC}$ & Overall \\
\hline \multicolumn{8}{|c|}{ Serum cortisol } \\
\hline To fasting & $15.9 \pm 1.4$ & $12.9 \pm 1.0$ & $11.7 \pm 1.1$ & NS & 0.013 & NS & 0.041 \\
\hline T30 & $15.1 \pm 1.6$ & $11.3 \pm 1.08^{*}$ & $11.7 \pm 0.8$ & - & - & - & 0.089 \\
\hline T60 & $13.3 \pm 1.5^{\star}$ & $11.5 \pm 0.7$ & $10.9 \pm 0.8$ & - & - & - & NS \\
\hline T120 & $12.9 \pm 1.3^{*}$ & $11.1 \pm 0.8$ & $8.8 \pm 0.7^{\star}$ & NS & 0.006 & 0.067 & 0.019 \\
\hline Nadir $^{\mathrm{a}}$ & $11.7 \pm 1.1^{*}$ & $9.9 \pm 0.7^{*}$ & $8.4 \pm 0.6^{*}$ & NS & 0.008 & NS & 0.028 \\
\hline AUC & $1679 \pm 159$ & $1383 \pm 83$ & $1280 \pm 73$ & - & - & - & 0.084 \\
\hline \multicolumn{8}{|c|}{ Plasma ACTH } \\
\hline TO fasting & $27.4 \pm 2.3$ & $26.0 \pm 2.1$ & $21.3 \pm 1.3$ & - & - & - & NS \\
\hline T30 & $23.6 \pm 3.0$ & $25.6 \pm 1.9$ & $22.4 \pm 1.4$ & - & - & - & NS \\
\hline T60 & $21.8 \pm 1.7$ & $29.8 \pm 3.1$ & $21.9 \pm 1.2$ & 0.017 & NS & 0.024 & 0.033 \\
\hline T120 & $23.2 \pm 1.7$ & $26.2 \pm 1.7$ & $19.6 \pm 1.2$ & 0.098 & - & 0.006 & 0.023 \\
\hline Nadir $^{\mathrm{a}}$ & $20.9 \pm 1.9$ & $24.2 \pm 1.6$ & $19.0 \pm 1.2^{*}$ & - & - & - & 0.074 \\
\hline AUC & $2689 \pm 262$ & $3286 \pm 247$ & $2563 \pm 135$ & - & - & - & 0.096 \\
\hline
\end{tabular}

${ }^{*} P<0.05$ compared to T0. $P<0.05$ are shown in bold.

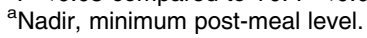




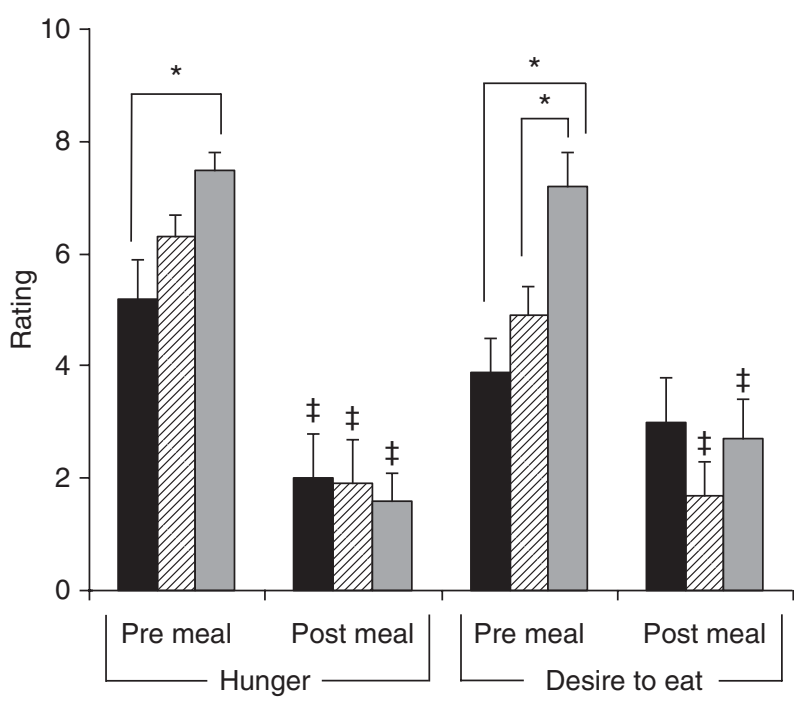

Figure 1 Pre- and post-meal appetite scores. Premeal hunger ratings were lower in AN than the other groups, and desire to eat was lower in AN and ANWR than HC. All groups reported significant decreases in hunger, and all but $A N$ reported a reduction in desire to eat after the meal. For post-meal, there were no significant between-group differences in hunger or desire to eat. ${ }^{\star} P<0.04$ compared with $\mathrm{HC} .{ }^{\ddagger} P<0.05$ vs premeal. Black, AN (active anorexia nervosa); striped, ANWR (weight-recovered anorexia nervosa); and grey, $\mathrm{HC}$ (healthy controls).

cortisol levels decreased after the meal. However, the post-meal decrease in ACTH levels was significant only in $\mathrm{HC}$, not in AN or ANWR.

\section{Fasting and post-prandial appetite ratings}

Subjective ratings of homeostatic (i.e. hunger) and hedonic (i.e. desire to eat favorite food) appetite using the visual analog scale are reported in Fig. 1. AN reported less hunger and lower desire to eat favorite foods and ANWR reported lower desire to eat favorite foods than $\mathrm{HC}$ in the fasting state $(P<0.04)$. In all groups, subjective hunger decreased after the meal. In ANWR and HC, but not AN, desire to eat favorite foods decreased after the meal. Post-prandial appetite ratings did not differ between groups.

\section{Relationship between HPA activation and appetite}

Associations between cortisol levels and subjective appetite are shown in Fig. 2. Across groups, fasting serum cortisol and cortisol AUC were negatively associated with subjective assessment of homeostatic (i.e. hunger) and hedonic (i.e. desire to eat favorite food) appetite in the fasting state. After controlling for BMI and depressive symptoms as assessed by BDI2 scores, these relationships remained significant.

\section{Relationship between cortisol levels and neurocircuitry involved in appetite and food motivation}

Table 2 shows how much of the variance in group differences in signal changes in brain activity associated with our significant food-motivation brain regions is related to cortisol levels, as measured by TO and AUC. We previously demonstrated premeal hypoactivation in AN (vs HC) in the hypothalamus, amygdala, hippocampus, OFC, and insula, and in ANWR (vs HC) in the hypothalamus, amygdala, and insula. We now demonstrate that cortisol levels, as assessed by TO and AUC, are associated with variance in activation in the hypothalamus (16-26\%), amygdala (24-45\%), hippocampus (20-23\%), OFC (19-46\%), and insula (11-12\%) in AN vs HC, and the hypothalamus (10$18 \%$ ), amygdala $(31-42 \%)$, and insula (10-12\%) in ANWR vs HC. After the meal, we reported decreased activation in AN (vs HC) in the amygdala and insula. Cortisol was associated with $13-16 \%$ of the betweengroup difference in amygdala activation. Finally, we previously found that post-meal activation in AN (vs ANWR) was increased in the amygdala and decreased in the insula. Cortisol was associated with $9-12 \%$ of between-group differences in brain activation in the insula. Controlling for BDI2 did not alter the results for $\mathrm{AN}$ vs HC, indicating that cortisol-brain relationships for this between-group contrast are independent of depressive symptoms in the AN group.

\section{Discussion}

Using a novel approach combining neuroendocrine, subjective appetite, and brain imaging assessments during the fasting state and in response to food, we show for the first time that HPA dysregulation in women with active and weight-recovered $\mathrm{AN}$ is associated with altered subjective appetite and food-motivation brain circuits. Importantly, these findings are independent of depressive symptoms. These data suggest that abnormalities in HPA secretory patterns are associated with altered perception of appetite, increasing the maintenance of AN symptoms, and, given findings in weight-recovered cases, represent a trait of this illness.

We found that cortisol levels were increased in women with active AN compared with healthy women during the fasting state and in response to a meal. Although postprandial cortisol levels have been studied in healthy individuals, with mixed results of increased, unchanged, or decreased levels following food intake $(28,29,30,31)$, there are little data on cortisol and ACTH patterns following a meal in AN. Gastric infusion of liquid caloric mixtures resulted in an increase in ACTH and cortisol in 15 women with AN compared with no change in 15 healthy women (31). To our knowledge, however, the effect of eating a meal 

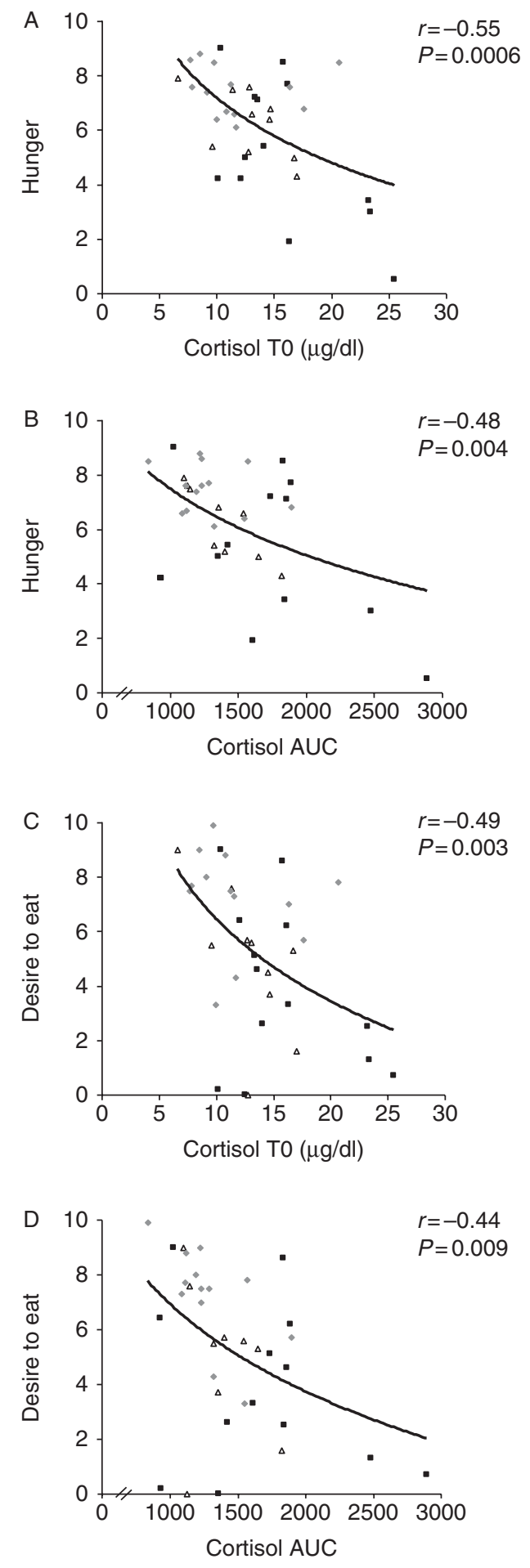

Figure 2 Cortisol levels and appetite. Fasting and AUC cortisol levels are inversely associated with fasting assessments of hunger ( $A$ and $B$ respectively) and desire to eat favorite foods ( $C$ and $D$ respectively). Squares, active anorexia nervosa; triangles, weight-recovered anorexia nervosa; and diamonds, healthy controls. on HPA hormones has not been reported in women with AN. In our study, cortisol levels decreased after the meal in all groups, while ACTH decreased in the healthy women only, suggesting excessive postprandial ACTH secretion in active NR and ANWR. The relatively higher cortisol levels that we report in women with active AN before and after a meal are consistent with the known HPA hyperactivation in this disorder $(1,2,3,4,5,6,7)$. We also found evidence of increased HPA drive in weight-recovered women with AN, who had higher postprandial ACTH levels compared with women with active AN and healthy women despite comparable cortisol levels. This is in line with the prior reports of persistent dysregulated HPA function in AN following weight gain $(8,9)$.

Reports of appetite are abnormal in $\operatorname{AN}(32,33)$. We report lower levels of subjective homeostatic (i.e. hunger) and hedonic (i.e. desire to eat favorite foods) appetite in women with active AN and lower levels of hedonic appetite in weight-recovered women with AN compared with healthy women. We now show that cortisol levels, as assessed by fasting cortisol or postprandial cortisol AUC, are negatively associated with self-reported homeostatic and hedonic appetite levels in AN, independent of BMI or depressive symptoms. Our data raise the question of whether HPA dysregulation, presumably driven by anorexigenic $\mathrm{CRH}$, may promote altered perception of appetite in AN.

Our previous work demonstrated hypoactivation of numerous regions of the brain involved in food motivation, including the hypothalamus (a key control center for appetitive signaling), amygdala (a region important for learning satiety cues and assessing the reward value of food), hippocampus (implicated in processing food-related memories), OFC (involved in integration of emotion and reward expectation), and insula (integrates visceral, homeostatic, and emotional signals) in women with AN compared with healthy women (16). Recent human neuroimaging studies have offered additional insight into the role of these regions, particularly in the interaction between appetite and stress in healthy populations. For example, acute stress has been shown to elicit variable responses to rewarding food stimuli in the amygdala and hippocampus, depending on appetite level and BMI classification $(34,35)$, with a significant association between basal cortisol and amygdala activation in response to palatable food (35). Moreover, the OFC appears to modulate the long-term effects of this interaction, with activation positively related to BMI only during stress (35). These findings demonstrate the dynamic interplay between HPA activation and food intake in these regions, although the effect of chronic stress on these systems is yet to be investigated.

Using a food-related fMRI paradigm, we previously showed that in response to viewing high-calorie foods compared with objects, premeal brain activation was decreased in women with active AN compared with 


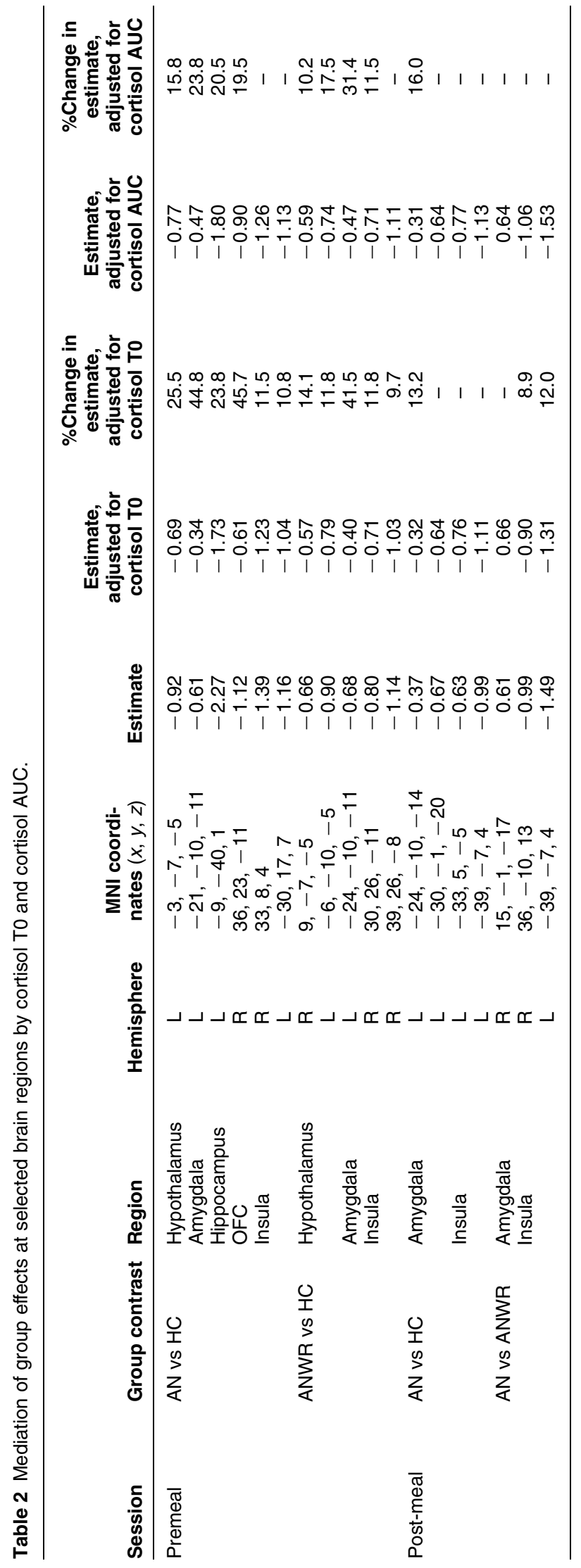

healthy women in the hypothalamus, amygdala, hippocampus, OFC, and insula (16). We now show that postprandial cortisol levels are associated with $16-46 \%$ of these between-group differences (HC vs AN) in activation in the hypothalamus, amygdala, hippocampus, OFC, and insula. Premeal brain activation was decreased in women with weight-recovered AN in the hypothalamus, amygdala, and insula (16). Cortisol levels are associated with $10-42 \%$ of between-group differences (HC vs ANWR) in activation in the hypothalamus, amygdala, and insula. After the meal, activation was decreased in women with active AN in the amygdala compared with healthy women and compared with healthy and weight-recovered women in the insula (16). Cortisol levels were associated with $4-16 \%$ of between-group differences in the activation of the amygdala and $9-12 \%$ of between-group differences in insular activation. Importantly in AN vs HC, these associations were independent of depressive symptoms as measured by the BDI2.

Overall, these results are consistent with the findings relating to acute HPA activation and response to food stimuli in the amygdala, hippocampus, and OFC. Importantly, they extend these previous results suggesting HPA disruption of appetitive signals to the effect of chronic stress and hypercortisolemia as a function of state (i.e. AN) as well as trait (ANWR). Given the density of CRF2 receptors, which (as opposed to CRF1 receptors) are particularly involved in appetitive signaling of CRF and downstream hormones (18), in these limbic and paralimbic regions (17), in combination with the evidence of disruption of HPA hormone levels in AN and ANWR, it is likely that the anorexigenic actions of CRH and orexigenic effects of cortisol influence activation of regions outside the hypothalamus and pituitary to influence hedonic and homeostatic perception of appetite in AN and ANWR.

Limitations of this study include small sample size, which may have reduced the power to detect betweengroup differences in endpoints, as well as correlations between HPA secretory patterns and appetite and brain activation. However, even with a relatively small sample size, our findings are robust. This is a cross-sectional study and causality cannot be established. Further research will be important to explore the effect of HPA dysregulation on appetite pathways in AN.

In summary, we provide evidence that dysregulation of HPA signaling in AN may be involved in disease pathogenesis. Fasting and postprandial cortisol levels are higher in women with active AN compared with healthy women, while postprandial ACTH levels are higher in women with weight-recovered AN compared with healthy women despite no significant difference in cortisol levels. Cortisol levels are negatively associated with homeostatic and hedonic measures of subjective appetite, independent of BMI and depressive symptoms. In addition, cortisol levels are associated with betweengroup differences in activation of brain regions 
involved in food motivation, independent of depressive symptoms. Together, these data suggest that HPA dysregulation is associated with the maintenance of AN symptoms, particularly given the findings in weightrecovered cases, through altered perception of appetite.

\section{Declaration of interest}

The authors declare that there is no conflict of interest that could be perceived as prejudicing the impartiality of the research reported.

\section{Funding}

This work was supported by the NIH (awards: UL1 RR025758, K12 HD051959, and K23 MH092560).

\section{Acknowledgements}

The authors thank the nurses and bionutritionist in the Massachusetts General Hospital Clinical Research Center and the subjects who participated in this study.

\section{References}

1 Misra M, Miller KK, Almazan C, Ramaswamy K, Lapcharoensap W, Worley M, Neubauer G, Herzog DB \& Klibanski A. Alterations in cortisol secretory dynamics in adolescent girls with anorexia nervosa and effects on bone metabolism. Journal of Clinical Endocrinology and Metabolism 200489 4972-4980. (doi:10.1210/ jc. 2004-0723)

2 Putignano P, Dubini A, Toja P, Invitti C, Bonfanti S, Redaelli G, Zappulli D \& Cavagnini F. Salivary cortisol measurement in normal-weight, obese and anorexic women: comparison with plasma cortisol. European Journal of Endocrinology 2001 145 165-171. (doi:10.1530/eje.0.1450165)

3 dos Santos E, dos Santos JE, Ribeiro RP, Rosa ESAC, Moreira AC \& Silva de Sa MF. Absence of circadian salivary cortisol rhythm in women with anorexia nervosa. Journal of Pediatric and Adolescent Gynecology 200720 13-18. (doi:10.1016/j.jpag.2006.10.011)

4 Gold PW, Gwirtsman H, Avgerinos PC, Nieman LK, Gallucci WT, Kaye W, Jimerson D, Ebert M, Rittmaster R, Loriaux DL et al. Abnormal hypothalamic-pituitary-adrenal function in anorexia nervosa. Pathophysiologic mechanisms in underweight and weight-corrected patients. New England Journal of Medicine 1986 314 1335-1342. (doi:10.1056/NEJM198605223142102)

5 Hotta M, Shibasaki T, Masuda A. Imaki T, Demura H, Ling N \& Shizume K. The responses of plasma adrenocorticotropin and cortisol to corticotropin-releasing hormone (CRH) and cerebrospinal fluid immunoreactive CRH in anorexia nervosa patients. Journal of Clinical Endocrinology and Metabolism 198662 319-324. (doi:10.1210/jcem-62-2-319)

6 Lawson EA, Donoho D, Miller KK, Misra M, Meenaghan E, Lydecker J, Wexler T, Herzog DB \& Klibanski A. Hypercortisolemia is associated with severity of bone loss and depression in hypothalamic amenorrhea and anorexia nervosa. Journal of Clinical Endocrinology and Metabolism 200994 4710-4716. (doi:10.1210/jc.2009-1046)

7 Lawson EA, Misra M, Meenaghan E, Rosenblum L, Donoho DA, Herzog D, Klibanski A \& Miller KK. Adrenal glucocorticoid and androgen precursor dissociation in anorexia nervosa. Journal of Clinical Endocrinology and Metabolism 200994 1367-1371. (doi:10.1210/jc.2008-2558)

8 Mayer L, Walsh BT, Pierson RN Jr, Heymsfield SB, Gallagher D, Wang J, Parides MK, Leibel RL, Warren MP, Killory E et al. Body fat redistribution after weight gain in women with anorexia nervosa. American Journal of Clinical Nutrition 200581 1286-1291.
9 Grinspoon S, Thomas L, Miller K, Pitts S, Herzog D \& Klibanski A. Changes in regional fat redistribution and the effects of estrogen during spontaneous weight gain in women with anorexia nervosa. American Journal of Clinical Nutrition 200173 865-869.

10 Britton DR, Koob GF, Rivier J \& Vale W. Intraventricular corticotropin-releasing factor enhances behavioral effects of novelty. Life Sciences 198231 363-367. (doi:10.1016/0024-3205(82) 90416-7)

11 Cavagnini F, Croci M, Putignano P, Petroni ML \& Invitti C. Glucocorticoids and neuroendocrine function. International Journal of Obesity and Related Metabolic Disorders 200024 (Suppl 2) S77-S79. (doi:10.1038/sj.ijo.0801284)

12 Heinrichs SC, Menzaghi F, Pich EM, Hauger RL \& Koob GF. Corticotropin-releasing factor in the paraventricular nucleus modulates feeding induced by neuropeptide Y. Brain Research 1993611 18-24. (doi:10.1016/0006-8993(93)91771-J)

13 Tataranni PA, Larson DE, Snitker S, Young JB, Flatt JP \& Ravussin E. Effects of glucocorticoids on energy metabolism and food intake in humans. American Journal of Physiology 1996271 E317-E325.

14 Tempel DL \& Leibowitz SF. Adrenal steroid receptors: interactions with brain neuropeptide systems in relation to nutrient intake and metabolism. Journal of Neuroendocrinology 19946 479-501. (doi:10.1111/j.1365-2826.1994.tb00611.x)

15 Lawson EA, Eddy KT, Donoho D, Misra M, Miller KK, Meenaghan E, Lydecker J, Herzog D \& Klibanski A. Appetiteregulating hormones cortisol and peptide YY are associated with disordered eating psychopathology, independent of body mass index. European Journal of Endocrinology $2011 \quad 164$ 253-261. (doi:10.1530/EJE-10-0523)

16 Holsen LM, Lawson EA, Blum J, Ko E, Makris N, Fazeli PK, Klibanski A \& Goldstein JM. Food motivation circuitry hypoactivation related to hedonic and non-hedonic aspects of hunger and satiety in women with active and weight-restored anorexia nervosa. Journal of Psychiatry \& Neuroscience 201237 322-332. (doi:10.1503/jpn.110156)

17 Chalmers DT, Lovenberg TW \& De Souza EB. Localization of novel corticotropin-releasing factor receptor (CRF2) mRNA expression to specific subcortical nuclei in rat brain: comparison with CRF1 receptor mRNA expression. Journal of Neuroscience 199515 6340-6350.

18 Chalmers DT, Lovenberg TW, Grigoriadis DE, Behan DP \& De Souza EB. Corticotrophin-releasing factor receptors: from molecular biology to drug design. Trends in Pharmacological Sciences 1996 17 166-172. (doi:10.1016/0165-6147(96)81594-X)

19 McEwen BS. Glucocorticoid receptors in the brain. Hospital Practice $1988114119-121$.

20 McEwen BS, De Kloet ER \& Rostene W. Adrenal steroid receptors and actions in the nervous system. Physiological Reviews $1986 \mathbf{6 6}$ 1121-1188.

21 McEwen BS, Weiss JM \& Schwartz LS. Uptake of corticosterone by rat brain and its concentration by certain limbic structures. Brain Research 196916 227-241. (doi:10.1016/0006-8993(69) 90096-1)

22 Lawson EA, Holsen LM, Santin M, Meenaghan E, Eddy KT, Becker AE, Herzog DB, Goldstein JM \& Klibanski A. Oxytocin secretion is associated with severity of disordered eating psychopathology and insular cortex hypoactivation in anorexia nervosa. Journal of Clinical Endocrinology and Metabolism 201297 E1898-E1908. (doi:10.1210/jc.2012-1702)

23 Lawson EA, Holsen LM, Santin M, DeSanti R, Meenaghan E, Eddy KT, Herzog DB \& Goldstein JM. Postprandial oxytocin secretion is associated with severity of anxiety and depressive symptoms in anorexia nervosa. Journal of Clinical Psychiatry 2013 74 e451-e457. (doi:10.4088/JCP.12m08154)

24 Diagnostic and Statistical Manual of Mental Disorders, 4th edn. DSMIV-TR. Washington, DC: American Psychiatric Association, 2000.

25 Frisancho AR \& Flegel PN. Elbow breadth as a measure of frame size for US males and females. American Journal of Clinical Nutrition 198337 311-314.

26 Flint A, Raben A, Blundell JE \& Astrup A. Reproducibility, power and validity of visual analogue scales in assessment of appetite 
sensations in single test meal studies. International Journal of Obesity and Related Metabolic Disorders 200024 38-48. (doi:10.1038/ sj.ijo.0801083)

27 Statistical Parameter Mapping: The Analysis of Functional Brain Images. Boston, MA: Academic Press, 2007.

28 Martens EA, Lemmens SG, Adam TC \& Westerterp-Plantenga MS. Sex differences in HPA axis activity in response to a meal. Physiology $\mathcal{E}$ Behavior 2012106 272-277. (doi:10.1016/j.physbeh.2012. 02.016)

29 Van Cauter E, Shapiro ET, Tillil H \& Polonsky KS. Circadian modulation of glucose and insulin responses to meals: relationship to cortisol rhythm. American Journal of Physiology $1992 \mathbf{2 6 2}$ E467-E475.

30 Alleman RJ Jr \& Bloomer RJ. Hormonal response to lipid and carbohydrate meals during the acute postprandial period. Journal of the International Society of Sports Nutrition 20118 19. (doi:10.1186/ 1550-2783-8-19)

31 Rigaud D, Verges B, Colas-Linhart N, Petiet A, Moukkaddem M, Van Wymelbeke V \& Brondel L. Hormonal and psychological factors linked to the increased thermic effect of food in malnourished fasting anorexia nervosa. Journal of Clinical Endocrinology and Metabolism 200792 1623-1629. (doi:10.1210/jc.2006-1319)
32 Halmi KA \& Sunday SR. Temporal patterns of hunger and fullness ratings and related cognitions in anorexia and bulimia. Appetite 199116 219-237. (doi:10.1016/0195-6663(91)90060-6)

33 Hetherington MM \& Rolls BJ. Eating behavior in eating disorders: response to preloads. Physiology \& Behavior 199150 101-108. (doi:10.1016/0031-9384(91)90505-I)

34 Born JM, Lemmens SG, Rutters F, Nieuwenhuizen AG, Formisano E, Goebel R \& Westerterp-Plantenga MS. Acute stress and food-related reward activation in the brain during food choice during eating in the absence of hunger. International Journal of Obesity 201034 172-181. (doi:10.1038/ijo. 2009.221)

35 Rudenga KJ. Sinha R \& Small DM. Acute stress potentiates brain response to milkshake as a function of body weight and chronic stress. International Journal of Obesity 201337 309-316. (doi:10.1038/ijo.2012.39)

Received 21 May 2013

Revised version received 8 August 2013

Accepted 14 August 2013 